ÜBER EINIGE FÜR PRIMZAHLEN CHARAKTERISTISCHE BEZIEHUNGEN

voN

\title{
JACOB HACKS
}

in CREFELD.

Es seien $m$ und $n$ zwei beliebige positive ganze Zahlen. [x] sei die grösste in $x$ enthaltene ganze Zahl. Stellt man dann die Gleichungen auf

$$
\begin{aligned}
m & =\left[\frac{m}{n}\right] n+r_{1}, \\
2 m & =\left[\frac{2 m}{n}\right] n+r_{2}, \\
\ldots & \ldots \ldots \\
(n-\mathrm{I}) m & =\left[\frac{(n-1) m}{n}\right] n+r_{n-1}
\end{aligned}
$$

und addirt, so kommt

$$
\frac{m n(n-1)}{2}=n \sum_{s=1}^{s=n-1}\left[\frac{s m}{n}\right]+\sum_{s=1}^{s=n-1} r_{s}
$$

Bezeichnet man jetzt mit $\partial$ den grössten gemeinschaftlichen Teiler von $m$ und $n$ und setzt $m=m^{\prime} \delta, n=n^{\prime} \delta$, so dass $m^{\prime}$ und $n^{\prime}$ relative Primzahlen sind, so ergibt sich leicht

$$
\sum_{i}^{n-1} r_{s}=\delta \frac{n^{\prime}\left(n^{\prime}-1\right)}{2} \delta=\frac{n\left(n-\delta^{\prime}\right)}{2} .
$$

Setzt man diesen Wert in (I) ein, so folgt

(3)

$$
\sum_{1}^{n-1}\left[\frac{s m}{n}\right]=\frac{(m-1)(n-1)}{2}+\frac{\delta-1}{2} .
$$

Acta mathomatica. 17. Imprimé le 3 mars 1893. 
Sind $m$ und $n$ relativ prim $\mathrm{zu}$ einander, so wird $\cdot \hat{o}=\mathrm{I}$ und

$$
\sum_{1}^{n-1}\left[\frac{s m}{n}\right]=\frac{(m-1)(n-1)}{2}
$$

Bezeichnet jetzt $p$ eine Primzahl, so ist $p$ relativ prim zu $1,2,3, \ldots, p-1$. Setzt man also in (4) $n=p$ und für $m$ der Reihe nach die Werte I , 2, 3, ..,p-1, so entstehen die Gleichungen

$$
\begin{gathered}
\sum_{1}^{p-1}\left[\frac{s}{p}\right]=0 \cdot \frac{p-1}{2}, \\
\sum_{1}^{p-1}\left[\frac{2 s}{p}\right]=1 \cdot \frac{p-1}{2}, \\
\sum_{1}^{p-1 !}\left[\frac{3 s}{p}\right]=2 \cdot \frac{p-1}{2}, \\
\ldots \ldots \\
\sum_{1}^{p-1}\left[\frac{(p-1) s}{p}\right]=(p-2) \frac{p-1}{2}
\end{gathered}
$$

durch deren Addition man die Beziehung erhält

$$
\sum_{y=1}^{y=p-1} \sum_{s=1}^{s=p-1}\left[\frac{y s}{y}\right]=\left(\frac{p-1}{2}\right)^{2}(p-2),
$$

welche ohne Anwendung von Summenzeichen folgende Gestalt hat:

$$
\begin{aligned}
& {\left[\frac{\mathrm{I}}{p}\right]+\left[\frac{2}{p}\right]+\left[\frac{3}{p}\right]+\ldots+\left[\frac{p-1}{p}\right] } \\
+ & {\left[\frac{2}{p}\right]+\left[\frac{4}{p}\right]+\left[\frac{6}{p}\right]+\ldots+\left[\frac{2(p-1)}{p}\right] } \\
+ & {\left[\frac{3}{p}\right]+\left[\frac{6}{p}\right]+\left[\frac{9}{p}\right]+\ldots+\left[\frac{3(p-1)}{p}\right] } \\
+ & \ldots \\
+ & {\left[\frac{p-1}{p}\right]+\left[\frac{2(p-1)}{p}\right]+\left[\frac{3(p-1)}{p}\right]+\ldots+\left[\frac{(p-1)(p-1)}{p}\right]=\left(\frac{p-1}{2}\right)^{2}(p-2) . }
\end{aligned}
$$

Diese Beziehung gilt nur für Primzahlen, wie sich leicht aus dem Umstande ergibt, dass nur eine Primzahl $p$ die Eigenschaft hat, relativ prim zu den Zahlen I , 2, ., p- I zu sein. Die Gleichung (5) ist also für Primzahlen charakteristisch. 
Über einige-für Primzahlen charakteristische Beziehungen.

Setzt man in (4) $m=p$ und $n$ der Reihe nach gleich $1,2,3, \ldots, p-\mathrm{I}$, so erhält man in derselben Weise die Gleichung

$$
\begin{aligned}
{\left[\frac{p}{2}\right]+\left[\frac{p}{3}\right]+\left[\frac{p}{4}\right]+\ldots+\left[\frac{p}{p-1}\right] } \\
+\left[\frac{2 p}{3}\right]+\left[\frac{2 p}{4}\right]+\ldots+\left[\frac{2 p}{p-1}\right] \\
+\left[\frac{3 p}{4}\right]+\ldots+\left[\frac{3 p}{p-1}\right] \\
\left.+\ldots+\frac{(p-2) p}{p-1}\right]=\left(\frac{p-1}{2}\right)^{2}(p-2),
\end{aligned}
$$

welche ebenfalls für eine Primzahl $p$ charakteristisch ist.

Eine ähnliche Gleichung erhält man aus der bekannten für zwei beliebige positive relative Primzahlen $m$ und $n$ gültigen Bezichung

$$
\sum_{1}^{\left[\frac{n}{2}\right]}\left[\frac{m s}{n}\right]+\sum_{1}^{\left[\frac{m}{2}\right]}\left[\frac{n s}{m}\right]=\left[\frac{m}{2}\right]\left[\frac{n}{2}\right]
$$

(Gauss' Werke, Band 2, S. 9), indem man $n$ gleich einer ungeraden Primzahl $p$ und für $m$ der Reihe nach die Werte $1,2,3, \ldots, p-\mathrm{I}$ setzt:

$$
\begin{aligned}
\sum_{1}^{\frac{p-1}{2}}\left[\frac{s}{p}\right]+\sum_{1}^{0}\left[\frac{p s}{\mathrm{I}}\right] & =0 . \frac{p-\mathrm{I}}{2}, \\
\sum_{1}^{\frac{p-1}{2}}\left[\frac{2 s}{p}\right]+\sum_{1}^{1}\left[\frac{p s}{2}\right] & =\mathrm{I} \cdot \frac{p-\mathrm{I}}{2}, \\
\frac{p-1}{2}\left[\frac{3 s}{p}\right]+\sum_{1}^{1}\left[\frac{p s}{3}\right] & =\mathrm{I} \cdot \frac{p-\mathrm{I}}{2}, \\
\sum_{1}^{\frac{p-1}{2}}\left[\frac{4 s}{p}\right]+\sum_{1}^{2}\left[\frac{p s}{4}\right] & =2 \cdot \frac{p-\mathrm{I}}{2}, \\
\frac{\sum_{1}^{2}}{\sum_{1}^{2}}\left[\frac{5 s}{p}\right]+\sum_{1}^{2}\left[\frac{p s}{5}\right] & =2 \cdot \frac{p-\mathrm{I}}{2}, \\
\sum_{1}^{p}\left[\frac{(p-1) s}{p}\right]+\sum_{1}^{2}\left[\frac{p s}{p-1}\right] & \cdot
\end{aligned}
$$


Durch Addition dieser Gleichungen ergibt sich

$$
\sum_{y=1}^{y=p-1} \sum_{s=1}^{s=\frac{p-1}{2}}\left[\frac{y s}{p}\right]+\sum_{y=1}^{y=p-1} \sum_{s=1}^{s=\left[\frac{y}{2}\right]}\left[\frac{p s}{y}\right]=\left(\frac{p-1}{2}\right)^{3} .
$$

Auch diese Gleichung ist für eine Primzahl $p$ charakteristisch, wie sich daraus ergibt, dass für zwei beliebige positive ganze Zahlen $m$ und $n$ mit dem grössten gemeinschaftlichen Teiler $\delta$ die Beziehung besteht

$$
\sum_{1}^{\left[\frac{n}{2}\right]}\left[\frac{m s}{n}\right]+\sum_{1}^{\left[\frac{m}{2}\right]}\left[\frac{n s}{m}\right]=\left[\frac{m}{2}\right]\left[\frac{n}{2}\right]+\left[\frac{\delta}{2}\right]
$$

(cf. Acta mathematica, Band Io, S. 34).

Die Gleichungen (5), (6) und (7) haben die Eigenschaft, dass sie ausser $p$ nur bestimmte Zahlen enthalten.

Für den grössten gemeinschaftlichen Teiler $\delta$ zweier Zahlen $m$ und $n$ ergeben sich aus (2), (3) und (8) bezw. folgende Ausdrücke:

$$
\begin{aligned}
& \grave{o}=\frac{n^{2}-2 \sum_{1}^{n-1} r_{s}}{n} ; \\
& \grave{o}=2 \sum_{1}^{n-1}\left[\frac{s m}{n}\right]-m n+m+n ; \\
& \grave{o}=2 \sum_{1}^{\left[\frac{n}{2}\right]}\left[\frac{s m}{n}\right]+2 \sum_{1}^{\left[\frac{m}{2}\right]}\left[\frac{s n}{m}\right]-2\left[\frac{m}{2}\right]\left[\frac{n}{2}\right]
\end{aligned}
$$

oder

$$
\hat{o}=2 \sum_{1}^{\left[\frac{n}{2}\right]}\left[\frac{s m}{n}\right]+2 \sum_{1}^{\left[\frac{m}{2}\right]}\left[\frac{s n}{m}\right]-2\left[\frac{m}{2}\right]\left[\frac{n}{2}\right]+1,
$$

je nachdem die Zahlen $m$ und $n$ beide gerade, oder wenigstens eine von ihnen ungerade ist. 